\title{
Myocardial Tissue Engineering
}

\author{
Tatsuya Shimizu \\ Institute of Advanced Biomedical Engineering \& Science \\ Tokyo Women's Medical University \\ Japan
}

\section{Introduction}

Many lives are lost due to heart diseases including myocardial infarction and cardiomyopathy. Recent reports have demonstrated that regenerative medicine has promising potential for recovering severe heart failure. Regenerative therapies for heart failure include cytokine, gene and cell therapy. Because many types of cardiovascular stem cells have been identified and their clinical potentials have been demonstrated for the past decade, cell injection therapy has most attracted both researchers and clinicians (Wollert 2008). On the other hand, significant cell loss due to washing out and cell death has become problematic in cell injection technique. So, as next generation of regenerative therapy for impaired heart, transplantation of myocardial patches fabricated by tissue engineering technology are emerging and are clinically applied. Furthermore, several challenges for fabricating functional myocardial tissues/organs, which are electrically communicated, pulsate synchronously and evoke contraction power, have also started (Zimmermann, Didie et al. 2006). These ambitious challenges may lead to reconstruction of malformed hearts and become alternative therapy for heart transplantation.

Heart tissues are composed of high-dense cylindrical cardiomyocytes and fibroblasts with abundant vascular network and collagen-based extracellular matrix (ECM). Cardiomyocytes pulsate via sodium and calcium ion transient through cell membrane. They are also electrically coupled by gap junctions composed of connexion 43 and rapid electrical propagation realizes simultaneous beating as a whole. Continuous blood flow supplies oxygen and nutrition, and withdraw the waste for high metabolic demand of heart tissues. These structure and function produce mechanical contractions as a blood pump. Therefore the researchers should take into account high density culture of cardiomyocyte and surrounding cells, sufficient micro blood vessel fabrication, cell/ECM orientation and proper cell-to-cell coupling for engineering heart tissues/organs.

Here, previous and current status of cell injection therapy, myocardial patch transplantation and pulsatile myocardial tissue fabrication is described with some future views.

\section{Cell injection therapy}

Cell injection therapy for damaged heart has been researched since the early 1990's. Many researchers have demonstrated the therapeutic potential of isolated cell transplantation into myocardium using various types of cell sources both in animal models and in some clinical 
trials (Puceat 2008). The mechanism of myocardial tissue regeneration has not been completely cleared, but most researchers have agreed that transplanted cells secrete several cytokines which promote neovascularization, prohibit fibrosis, decrease cell death and recruit stem cells, leading to heart function improvement. It has been also asserted that some of injected cells differentiate into functional cardiomyocytes and may directly contribute to heart contraction improvement. Although some differences may exist in according to cell types, multifactorial mechanisms seem to relate with myocardial tissue regeneration.

In addition to cell sourcing, different routes are used for cell administration. Systematic intravenous infusion is performed through a central or peripheral vein. This method is simple and less invasive, however widespread distribution cause low ratio of cell engraftment. Most popular approach is intracoronary cell infusion via a balloon-catheter. Injected cells are reached directly in the target myocardial region, however, cells have to transmigrate across endothelium wall. Intracardiac injection is performed via pericardium during open heart surgery and via endocardium by a catheter with a 3-D electromechanical mapping system (NOGA mapping system). These methods realize relatively targeted delivery, but myocardial damage and arrhythmia induction are problematic. Future clarification will be needed to decide which is the best approach for cell injection.

\subsection{Skeletal myoblasts}

Skeletal myoblasts were the first cell source to enter the clinical application for heart tissue repair. They lie in a quiescent state on the basal membrane of myofibers and have the potential to start to proliferate and differentiate into functional skeletal muscle in response to muscle damage. They can be isolated autologously and be expanded from a single biopsy. In addition, skeketal myoblasts are relatively resistant to ischemia. Menasche and colleagues first applied skeletal myoblast injection via epicardium for patients undergoing coronary artery bypass grafting (CABG) (Menasche, Hagege et al. 2001). The phase I clinical study (MAGIC I) have shown the feasibility of skeletal myoblast implantation, however, increased risk of ventricular arrhythmias after the operation. Then, MAGIC II trial was performed to clarify the safety and efficacy, in which all patients received preventive medication and an implantable cardioverter-defibrillator for rescuing critical ventricular arrhythmias. In result, skeletal myoblast injection failed to significantly improve heart function, leading to sample size reduction (Menasche, Alfieri et al. 2008). On the other hand, the trial indicated the possibility that high dose cell injection might recover left ventricular dilatation. In addition, the other clinical trials of catheter-based myoblast implantation via endocardium have revealed functional efficacy (Opie and Dib 2006). According to these results, not the regenerative potential of myoblasts themselves but the amount of injected cells and delivery system may affect the efficacy. Therefore, it seems that skeletal myoblasts should not be excluded as a cell source for heart tissue repair. More optimization of cell delivery and comparison of cell sources can address these critical issues.

\subsection{Bone marrow-derived cells}

Bone marrow-derived cells are the most used cells in clinical trials for myocardial tissue repair (Wollert 2008). The discovery of circulating progenitor cells originated from human bone marrow has stimulated research and clinical use of bone marrow-derived cells 
(Asahara, Murohara et al. 1997). Bone marrow cells contain different stem and progenitor cells which will differentiate into various types of cells including endothelial cells, smooth muscle cells and cardiomyocytes. Bone marrow mononuclear cells (BMNCs), which can be isolated simply by gradient sedimentation after bone marrow aspiration without culture expansion, have been clinically injected via coronary artery from the first. BMNCs include heterogeneous cell population of monocytes, hematopoietic stem cells and endothelial progenitor cells (EPCs). Therefore, some groups have used BMNCs selected by surface markers $\left(\mathrm{CD} 34^{+}, \mathrm{CD} 133^{+}\right)$and demonstrated more efficacy of their injection. As another cell population, mesenchymal stem cells (MSCs) have been researched and clinically used. Although, MSCs represent between 0.01 and $0.001 \%$ of all nucleated cells in bone marrow, they can be readily expanded in culture. MSCs have the potential to differentiate into various types of cells and injected MSCs in heart seem to differentiate into myocardial composing cells. Recent studies have revealed rare happening of cardiomyocyte differentiation, therefore MSCs seem to recover heart function via their cytokine secretion and partial differentiation into vascular cells. As a unique feature, MSCs have the potential to escape from immune detection due to the direct inflammatory inhibition and the lack of cell-surface molecules. This property has realized allogenic mesenchymal stem cell transplantation in clinic and has given high impact on cell therapy research field.

Recent randomized controlled trials of bone marrow-derived cell injection revealed overall feasibility and safety. However the data has revealed only marginal increases of ejection fraction (EF) even in positive studies (0-5\%) (Martin-Rendon, Brunskill et al. 2008). For establishing more effective bone marrow-derived cell therapy, optimization of cell source, cell dose, delivery method and deliver timing will be needed.

\subsection{Adipose-derived stem cells}

In addition to bone marrow-derived MSCs, stem cells isolated from the stroma of adipose tissues have represented regenerative potential for heart tissues (Psaltis, Zannettino et al. 2008). Adipose tissue-derived stem cells (ASCs) display features similar to that of bone marrow-derived MSCs and their angiogenic potential have been reported. Some studies have also revealed cardiomyocyte differentiation from ASCs. It has not been clarified which mesenchymal stem cells are superior to other cell types, however, reatively easy isolation of adipose tissue may push the clinical application of ASCs.

\subsection{Cardiac stem cells}

Cardiac stem cells (CSCs) are also possible cell source for myocardial tissue regeneration. Two groups first reported CSC existence in 2003 (Beltrami, Barlucchi et al. 2003; Oh, Bradfute et al. 2003). Until then, it was common knowledge that heart was a post mitotic organ, but those reports accelerated the researches for identifying surface marker of CSCs and culturing them. Islet-1, Sca-1 and c-kit have been known as CSC markers. Recently, it has been also confirmed that heart has renewal ability at normal state and the annual rate of turning over is $1 \%$ at the age of 25 (Bergmann, Bhardwaj et al. 2009). Although the ability of CSCs may increase after heart injury, newly formed cardyomyocytes are not sufficient for replacing damaged muscle tissues. Therefore isolation and expansion of CSCs have been extensively examined. Some groups have used a different approach to make cardiospheres from biopsied myocardium, which lead to efficient CSC expansion (Lee, White et al. 2011). 
Clinical trials for injection therapy of autologous CSCs isolated from biopsy sample are now on going.

\subsection{Embryonic stem cells}

Although abundant studies demonstrated that MSCs, ASCs and CSCs have the potential of cardiomyocyte differentiation regarding gene and protein expression, there are no studies clearly showing beating cardiomyocytes differentiated from those stem cells. On the other hand, many researchers have confirmed that embryonic stem cells (ESCs) can differentiate into beating cardiomyocytes in vitro and implantation of ESC-derived cardiomyocytes improves damaged heart function. Several signal pathways for cardiac differentiation have been already clarified and various molecules have been reported as its promoters. For example, noggin increased cardiac differentiation efficacy via regulation of Bone morphogenetic protein (BMP) signalling pathway (Yuasa, Itabashi et al. 2005) and insulinlike growth-factor-binding protein 4 (IGFBP4) promotes cardiogenesis by inhibitor of canonical Wnt signalling (Zhu, Shiojima et al. 2008). In addition, fibloblast growth factor (FGF), retinoic acid, ascorbic acid and cyclosporine A have been reported to have the potential to enhance cardiac differentiation from ESCs. The important issue as well as cardiac differentiation is purification of cardiomyocytes from heterogeneous cell mixture, because contamination of immature cells leads to teratoma formation. Although genemodified ESCs harboring neomycin resistance gene or green fluorescent protein (GFP) gene in the cardiac-specific gene locus are very useful in non-clinical experiments, safe and efficient isolation technologies will be needed for clinical application. Culture media control focusing on the differences of cell metabolism may be useful for safe cell selection. Moreover immune response of the host is another critical issue. Nucler transfer or cell banking is possible approach avoiding immunoreaction.

Electrical communication and simultaneous beating of implanted ESC-derived cardiomyocytes should be also requested for improving damaged heart function without arrhythmia. In vivo electrophisiological analyses and the transplantation technology for synchronization will be essential for clinical application of these cells.

\subsection{Induced pluripotent stem cells}

Induced pluripotent stem cells (iPSCs) also hold great promise for myocardial tissue engineering (Vunjak-Novakovic, Tandon et al. 2010). Terminally differentiated cells can be reprogrammed to have the same potential as ESCs by introducing 3 or 4 transcriptional factor genes. Furthemore none-gene transfer technologies have been developed in the world. The superiority of iPSCs to ESCs is autologous cells, which do not cause immune response. Cardiac differentiation of human iPSCs has been reported in the same manner with ESCs.

Several critical issues must be clarified for clinical use, but ESCs/iPSCs-derived cardiomyocytes should contribute to myocardial tissue engineering in the view point of their pulsatile function and scaling-up.

\subsection{Problems of cell injection therapy}

Cell injection therapies for heart failure are now world-widely performed. While moderate success of direct cell injection has been observed, the efficacies seem not to reach the level that general clinicians think cell therapy a reliable treatment for heart failure. More 
optimization of cell source, cell preparation process, injection route, injection timing and patient population may increase the effectiveness; however one of the essential issues is cell delivery methodology. Cell injection therapy has significant difficulties about cell retention in the target tissue. The shape, size, and position of the grafted cells are often uncontrollable and large amount of the cells are washed-out. Moreover, once retaining cells die due to necrosis and apoptosis. Time course quantification with TUNEL assay demonstrated that a large number of the grafted cells die within a few days after injection in rat models (Zhang, Methot et al. 2001). In the clinical trial using bone marrow-derived cells, it has been also demonstrated that only $1-3 \%$ of the cells infused via coronary arteries could be detected by 3D positron emission tomography (PET) imaging of the patient heart. In this study, a large percentage of cells were found in the liver and spleen immediately after the procedures (Hofmann, Wollert et al. 2005). To clear the problem of cell loss, hydrogel-cell mixture injection has been pursued. Fibrin, collagen and alginate hydrogels are now used. Hydrogels with cells are injected as a liquid phase through syringe or catheter, then, they are polymerized and fixed in the target tissues (Kofidis, de Bruin et al. 2004). In hydrogel-cell mixture injection therapy, local tissue damage due to space occupation of hydrogel itself and inflammatory reaction due to hydrogel biodegradation are problematic.

Therefore, more advanced cell delivery systems have been requested to spread the regenerative therapy as one of the reliable treatments for heart failure.

\section{Tissue engineering}

Recent advance of tissue engineering technologies have realized the transplantation of tissue-engineered construct "myocardial patch" covering over damaged heart surface instead of simple cell injection into myocardium. Grafted cells within myocardial patches can survive more and secrete more cytokines, resulting in more heart function improvement. Furthermore pulsatile myocardial tissues have been successfully engineered by using cardiomyocytes as a seeding cell source. These tissues may directly help heart contraction and total heart wall replacement may be possible in future. There are several contexts of tissue engineering.

\subsection{Scaffold-based tissue engineering}

Most popular technology of tissue engineering is to seed cells into 3-D pre-fabricated biodegradable scaffolds which are made from synthetic polymer and biological material. Hydrogel formation after mixing cells and scaffold solution is another approach. Decellularized tissues have been also used as scaffolds. These scaffolds play as alternatives for extra cellular matrix (ECM), therefore, their cell-adhesiveness and porosity affect survived cell amount and engineered tissue quality. Scaffold modification can control its biodegradation and tissue formation. Growth factor linkage leads to accelerating tissue formation. Now these scaffold-based tissue engineering has been widely applied to cardiovascular tissue regeneration as well as other tissue repair (Vunjak-Novakovic, Tandon et al. 2010).

\subsection{Cell sheet-based tissue engineering}

In contrast to scaffold-based tissue engineering, our group have developed unique technique involving cell sheet stacking to fabricate 3-D tissues (Shimizu, Yamato et al. 2003). 
Cell sheets are 2-D connecting pure cells without any scaffolds, therefore cell-dense 3-D tissues can be fabricated by stacking cell sheets. Cell sheets are harvested from intelligent culture surface "temperature-responsive culture surface", which are covalently grafted with temperature-responsive polymer, poly ( $\mathrm{N}$-isopropylacrylamide) (PIPAAm) (Okano, Yamada et al. 1993). The surfaces are slightly hydrophobic and cell-adhesive at $37^{\circ} \mathrm{C}$, on the other hand, the surface changes to hydrophilic and not cell adhesive below $32^{\circ} \mathrm{C}$. Confluently cultured cells on the surface can detach as a contiguous cell sheet simply by reducing temperature. Furthermore, biological molecules underneath cell sheets are also preserved and play a critical role as an adhesive agent during cell sheet stacking. Cell sheet-based tissue engineering has been applied for a wide range of regenerative medicine including corneal epithelial replacement, heart tissue repair, pneumothorax repair, liver tissue repair and so on.

According to the spread of the concept fabricating 3-D tissues from 2-D confluent cells, several other technologies using this concept have emerged. Cell sheet fabrication techniques using fibrin coated dishes or nanofibrous polycaprolactone meshes have been reported (Shin, Ishii et al. 2004; Itabashi, Miyoshi et al. 2005). Cell sheet-like constructs have been also engineered using magnetite nanoparticles (Ito, Hibino et al. 2005). Magnetically labelled cells are attached on culture materials by magnetic force and confluent cells are harvested as a cell sheet by magnetic force release. Thus, cell sheet-based tissue engineering has now spread in the world as scaffold-free tissue engineering.

\section{Myocardial patch transplantation}

Both scaffold-based and cell sheet-based tissue engineering have been used for myocardial patch fabrication. Not only cardiomyocytes but also other types of cells have been used for creating myocardial patches and some myocardial patches using non-cardiomyocytes have been already clinically transplanted over damaged hearts. (Fig. 1.)

\subsection{Scaffold-based mayocardial patch}

In myocardial patch fabrication, synthetic polymer, biological material and decellularized tissue have been used as prefabricated scaffolds. Li and colleagues, who were one of the pioneer groups of myocardial tissue engineering, first demonstrated that gelatine sponges seeded with cardiac cells have therapeutic potentials for cryoinjured rat hearts ( $\mathrm{Li}$, Jia et al. 1999). Leor and colleagues reported that bioengineered heart grafts using porous alginate scaffolds attenuated left ventricular dilatation and heart function deterioration in infarction model (Leor, Aboulafia-Etzion et al. 2000). Eschenhagen and Zimmermann's group have developed innovative myocardial tissue engineering approach (Zimmermann, Schneiderbanger et al. 2002). They have fabricated 3-D tissues by gelling mixture of cardiac cells and collagen solution. The constructs induced systolic wall thickening of the left ventricle infracted area and improved fractional shortening of damaged hearts in rat myocardial infarction model (Zimmermann, Melnychenko et al. 2006). Small intestinal submucosa (SIS) has also been used as a scaffold for myocardial patch. MSC-seeded SIS improved heart contraction in rabbit infarction model (Tan, Zhi et al. 2009). There have been various types of myocardial patches using different scaffolds and different cell sources. Although implantable human myocardial patches using beating cardiomyocytes have not been established now, clinical trials of collagen-based myocardial patch with bone marrow 
cells (MAGNUM trial) (Chachques, Trainini et al. 2007) and vicryl mesh-based myocardial patches with fibroblasts (Anginera) ((Mirsadraee, Wilcox et al. 2006)) have revealed feasibility and safety of myocardial patch transplantation.

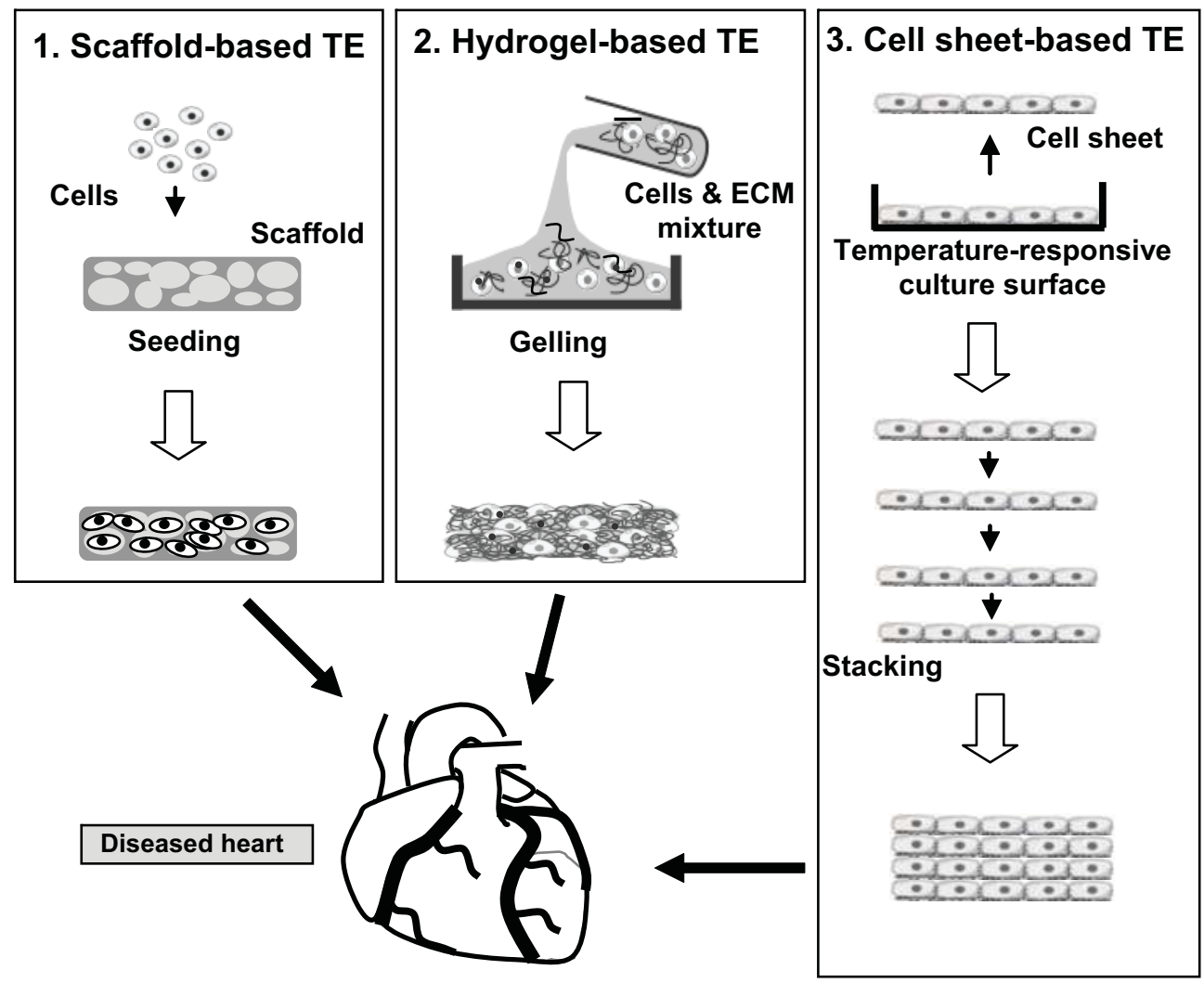

Fig. 1. Tissue engineering (TE) strategies for myocardial patch fabrication

\subsection{Cell sheet-based myocardial patch}

Many types of cell sheets have been reported to improve impaired heart function (Shimizu, Sekine et al. 2009). Cell sheets are transplanted onto heart surface directly via open heart surgery and cells can be more effectively delivered as thin, but large-area cell-dense grafts than isolated cell injection. Scaffold-based myocardial patches are usually transplanted on myocardium with suture, on the other hand, cell sheets are transplanted with no suture because biological adhesive proteins underneath cell sheets promote the attachment. When neonatal rat cardiac cell sheets were transplanted onto infracted rat hearts, grafted cardiomyocytes communicated with host myocardium via gap junctions and blood vessels formed within the graft, resulting in significant improvement of heart function (Miyagawa, Sawa et al. 2005; Sekine, Shimizu et al. 2006).

Sawa and colleagues have started to use skeletal myoblasts for cell sheet fabrication, because myoblasts can be isolated autologously and are relatively resistant to ischemic condition. 
The recovery of heart function by skeletal myoblast transplantation has been confirmed in rat ischemic model, in dilated cardiomyopathy hamster model, in pacing-induced canine heart failure model and in pig infarction model (Memon, Sawa et al. 2005; Hata, Matsumiya et al. 2006; Kondoh, Sawa et al. 2006; Miyagawa, Saito et al. 2010). Regarding stacking cell sheet number, 3-5 layers are optimal and more layering cause primary necrosis of the constructs (Sekiya, Matsumiya et al. 2009). They have demonstrated more hematopoietic stem cells and less fibrosis in cell sheet transplantation than in isolated cell injection in accordance with more expression of stromal-derived factor 1 (SDF-1), hepatocyte growth factor (HGF), and vascular endothelial growth factor (VEGF). Based on these results, clinical trial of autologous myoblast sheet transplantation for severe heart failure has started and the detailed results will appear soon.

In the same manner with cell injection therapy, MSCs are used as a candidate cell source for human implantable cell sheet. Adipose tissue-derived MSCs and menstrual blood-derived MSCs have improved damaged heart function in rat infarction model (Miyahara, Nagaya et al. 2006; Hida, Nishiyama et al. 2008). MSCs can gradually grow to form a thick stratum containing newly formed blood vessels and some cells seem to differentiate into cardiomyocytes at least by histological analyses. Further studies will be needed to confirm the differentiation into functional beating cardiomyocytes and possibilities to differentiate into unexpected cell types.

As emerging cell source, cell sheets of stem cell antigen 1-positive (Sca-1-positive) CSCs ameliorates cardiac dysfunction in mouse infarction model through cardiomyocyte differentiation and paracrine mechanisms mediated via soluble vascular cell adhesion molecule 1 (VCAM-1)/very late antigen-4 (VLA-4) signaling pathway (Matsuura, Honda et al. 2009). In addition, cardiac cell sheets originated from ESCs/iPSCs have been successfully fabricated and their transplantation into animal models is now ongoing.

For enhancing the efficacy of cell sheet transplantation, gene-modified cell sheets have been examined. Bcl-2 expressed myoblast sheets prolonged survival, increased production of proangiogenic paracrine mediators, and enhanced the therapeutic efficacy (Kitabayashi, Siltanen et al. 2010). HGF overexpression in myoblast sheets enhances their angiogenic potential in rat chronic heart failure model (Siltanen, Kitabayashi et al. 2011). As another concept, cell sheets co-cultured with endothelial cell sources have been transplanted in rat infarction models. Transplantation of EPC co-cultured fibroblast sheet improved heart function more than only fibroblast sheet implantation or EPC injection (Kobayashi, Shimizu et al. 2008). Furthermore, endothelial cell co-culture within cardiomyocyte sheets induced more neovascularization and more improvement of cardiac function than only cardiomyocyte sheets (Sekine, Shimizu et al. 2008). These studies indicate advanced strategies of cell sheet transplantation.

As mentioned previously, it is considered that the main mechanism of heart function improvement is neovascularization, fibrosis inhibition, apoptosis inhibition and stem cell recruitment due to various cytokines secreted from grafted cells. In comparison with cell injection approach, increase of cell survival within myocardial patches leads to more cytokine secretion, then, resulting in more function improvement. In addition to cytokine secretion, myocardial patches may have girdling effect and prohibit heart dilatation. Therefore, myocardial patch transplantation is quite different cell delivery method from cell injection and has more potential to rescue diseased hearts. In the case of myocardial patches using beating cardiomyocytes, direct enhancement of contraction power is additionally 
expected, however, electrical synchronization between host hearts and transplanted patches is a critical issue to be clarified.

\section{Engineering plusatile myocardial tisssue}

Beyond myocardial patch fabrication, several research groups have challenged to fabricate pulsatile myocardial tissues by their original tissue engineering strategies. Bioengineered contractile myocardial tissues may realize new therapeutics for severe heart diseases and be useful as alternatives for animal models.

\subsection{Pre-fabricated scaffold-based myocardial tissue fabrication}

The first approach for engineering functional myocardial tissue is seeding cardiomyocytes into synthetic or biological 3-D scaffolds. Vunjak-Novakovic and colleagues first reported that seeding primary cultured cardiomyocytes onto disc-shaped polyglycolic acid (PGA) scaffolds in rotating bioreactor system resulted in spontaneously pulsatile myocardial tissues (Papadaki, Bursac et al. 2001). Optimization of cell population, serum concentration and scaffold coating improved electrical conduction velocity of engineered constructs. Radisc and colleagues seeded rat cardiomyocytes in Matrigel onto collagen sponges and stimulated the constructs electrically. The stimulation improved the conductive and contractile properties in accordance with increased expression of myosin heavy chain and connexion 43. Furthermore, cardiomyocytes in the electrically stimulated constructs were more aligned and elongated as same as those in native heart tissue (Radisic, Park et al. 2007).

Following these studies, many research groups have started to engineer myocardial tissue in vitro by using various types of scaffolds. Scaffold porosity is one of the critical factors for pre-fabricated scaffold-based tissue engineering. High porosity increases seeded cell number and facilitates mass transport. Surface modyfication is also important for cell attachment and survival. Laminin coating improved cardiomyocyte adhesiveness. In addition, scaffold elasticity and degradability affect contraction property of engineered myocardium. Further studies are ongoing to development appropriate scaffold materials for myocardial tissue engineering.

\subsection{Hydrogel-based myocardial tissue fabrication}

The second approach is to form 3-D tissues by gelling of cardiac cell and matrix solution mixture. Eschenhagen and Zimmermann have continuously developed this strategy using collagen gel and successfully engineered macroscopically beating cardiac tissues (Zimmermann and Cesnjevar 2009). First, neonatal rat cardiomyocytes were suspended in collagen I solution and the mixture was poured into the mold. After gelling, the constructs were unidirectionally stretched with the mechanical devise. They have also realized contraction force measurement. Cyclic stretch introduced cell alignment along the stretching direction and increased mitochondrial density, leading to native heart-like tissue. The contraction force of engineered myocardium was comparative with native heart tissue and responded to pharmacological agents properly. Ring-shaped myocardial tissues were also fabricated and combined 5 constructs were transplanted onto infarcted rat hearts. Interestingly, the constructs synchronized to each other and improved damaged heart function. They have also confirmed that co-culture constructs including cardiomyocytes, 
fibroblasts and endothelial cells were superior to cardiomyocyte rich constructs in morphology and function. Recently, they have also started to utilize human cardiomyocytes differentiated from ESC/iPSC as cell source and challenged to create human myocardial tissues (Zimmermann 2011). In contrast to pre-fabricated scaffold usage, relatively homogeneous myocardial tissues are engineered by hydrogel-based approach. Therefore collagen gel-based myocardial tissue engineering has now become popular in the world.

\subsection{Cell sheet-based myocardial tissue fabrication}

The third approach is to engineer 3-D pulsatile myocardial tissues by stacking cardiac cell sheets. As mentioned previously, 2-D cell sheets can be harvested from temperatureresponsive culture dishes only by lowering temperature and do not include any materials. 3$\mathrm{D}$ tissues are constructed by layering cell sheets. Because 2-D confluent cells are directly stacked without any scaffolds, resulting constructs are cell-dense 3-D tissues. It is wellknown that 2-D confluent cardiomyocytes connect to each other electrically via gap junctions resulting in synchronized beating. Cardiac cell sheets harvested from temperatureresponsive culture dishes maintain this synchronized pulsation (Shimizu, Yamato et al. 2002). For creating 3-D functional heart tissues by layering cardiac cell sheets, morphological and electrical communications between cell sheets are critical. Multiple-electrode extracellular recording system revealed that double-layer rat cardiac cell sheets coupled electrically about one hour after layering and histological analysis showed the existence of connexin 43 between two cardiac cell sheets. Adhesive proteins deposited on cell sheet surface are considered to promote these rapid electrical communications (Haraguchi, Shimizu et al. 2006). Stacked cardiac cell sheets beat synchronously in macroscopic view and the constructs transplanted into rat subcutaneous tissues also pulsated continuously at least up to one year and eight months after implantation. Morphological analyses showed elongated cardiomyocytes, well-differentiated sarcomeres, gap junctions and multiple blood vessels, which were characteristic structure of native heart tissue (Shimizu, Yamato et al. 2002). Long-term observation revealed that their size, conduction velocity, and contractile force increased in proportion to the host growth (Shimizu, Sekine et al. 2006). Recently, fabrication of cardiac cell sheets using ESC-originated cardiomyocytes have just started and human cardiac cell sheets will appear in near future.

\subsection{Fabrication of vascularized myocardial tissue}

One of the major obstacles in myocardial tissue engineering is scaling-up of the constructs. Insufficient supply of oxygen and nutrient, and waste accumulation limit their thickness. Actually, cells are sparse in the central area, on the other hand, cells are dense in the outer surface $(100-200 \mu \mathrm{m})$ area in scaffold-based myocardial tissue engineering. In the case of cell sheet-based myocardial tissue engineering, thickness limit is approximately $80 \mu \mathrm{m}$ (3 layers) (Shimizu, Sekine et al. 2006). Several approaches have been examined in the point of view overcoming diffusion limit. Perfusion of culture media through the constructs using porous scaffolds is one possible approach. Media penetration increased cell migration depth and improved cell metabolism. However shear stress due to media flow may prohibit tight cell attachment on the scaffold material. Media perfusion with oxygen carrier, perfluorocarbon (PFC) has been also examined for improving oxygen transport. PFC usage increased cell proliferation and improved pulsatile function. Media penetration is useful to some extent, however, it becomes more difficult as cell density increases. 
To overcome this problem, it has been requested to develop new technologies for introducing vasculature or vascular-like structure into engineered tissues. Several researchers have tried to generate microchannel network within porous 3-D scaffolds by microfabrication techniques including $\mathrm{CO}_{2}$ laser ablation. The technology has not reached to mimicking native micro capillary network. On the other hand, recent studies have revealed that co-cultured endothelial cells within cardiac constructs can spontaneously form vascular-like network in vitro and tubular formation has been found in some parts. It has been also confirmed that this pre-vascular structure connected to host blood vessels immediately after transplantation and the newly developed vessels within the constructs were blood-supplied within a few days (Sekiya, Shimizu et al. 2006). We have already demonstrated that the tissue thickness of cardiac cell sheets co-cultured with endothelial cells were just twice as the thickness of cardiac cell sheets without endothelial cells (Sekine, Shimizu et al. 2008). Although endothelial cell co-culture is helpful for accelerating blood vessel formation, more scaling-up is still limited due to primary ischemia until sufficient vascularization.

One possible idea for scaling-up is utilizing in vivo vascularization power. Our group has reported that triple-layer cardiac cell sheets were repeatedly implanted after waiting enough vascular formation within previously implanted tissues. In result, synchronously beating thick myocardial tissues with sufficient micro capillaries were successfully fabricated and 10-times transplantation of triple-layer constructs (totally 30 sheets) formed 1-mm thick, pulsatile myocardial tissues. Furthermore, when triple-layer grafts were transplanted repeatedly over a surgically connectable artery and vein in leg, the multilayer constructs were blood-supplied from the thick artery and vein. The constructs were successfully resected with the connectable blood vessels and were ectopically transplanted in neck with direct vessel anastomoses (Shimizu, Sekine et al. 2006). Recently several groups have also utilized in vivo power for myocardial tissue engineering. Cardiomyocytes, ECM alternatives and native blood vessels were packed in the special chamber and incubated in vivo. Vascularized heart-like tissues were created in the body (Morritt, Bortolotto et al. 2007; Birla, Dhawan et al. 2009).

Furthemore, next challenge is now in vitro fabrication of vascularized myocardial tissues. Kofidis and colleagues have constructed fibrin gel-based myocardial tissues containing rat aortas (1-2mm), through which culture media was perfused (Kofidis, Lenz et al. 2003). Cell survival and metabolism were improved, however formation of functional blood vessels connecting with central aortas were not clear. We are now trying to promote endothelial cell tubular formation within in vitro engineered cardiac tissues and to perfuse culture media through the newly formed vessels using perfusion bioreactors. Further studies will be needed to break through the obstacles for in vitro scaling up.

\subsection{From tissue engineering to organ engineering}

For future organ engineering, some groups have challenged to engineer myocardial constructs with pumping function. Ott and colleagues have used decellularized organ as a scaffold. They decellularized rat whole hearts and re-seeded cardiac cells into decellularized hearts. Heart contraction was recovered and pump function was generated (Ott, Matthiesen et al. 2008). Zimmermann's group developed pouch-like myocardial tissue by their technology as previously described and covered heart with pouch-like constructs (Yildirim, Naito et al. 2007). 
Regarding cell sheet technology, myocardial tubes have been fabricated by wrapping rat cardiac cell sheets around fibrin tubes and rat resected aortas. The engineered myocardial tubes revealed spontaneous, synchronized pulsation and small but significant inner pressure changes (about $0.1 \mathrm{mmHg}$ ) in vitro (Kubo, Shimizu et al. 2007). On the other hand, resected rat aortas wrapped with cardiac cell sheets were micro surgically transplanted in place of the abdominal aorta. After 1 month, in vivo myocardial tubes demonstrated spontaneous beating and evoked independent blood pressures (about $6 \mathrm{~mm} \mathrm{Hg}$ ). The value of in vivo myocardial tubes was much bigger than in vitro myocardial tubes (Sekine, Shimizu et al. 2006). Comparing in vitro and in vivo, it was considered that pulsation due to host blood flow has induced cardiomyocyte hypertrophy, leading to improvement of pumping function. Therefore pulsatile perfusion bioreactors may improve pumping function of in vitro engineered myocardial tubes.

Thus, small size myocardial constructs evoking pumping function have been realized. Expansion and selection of cardiomyocytes, and sufficient blood vessel formation for scaling-up are now critical issues for organ engineering.

\section{Conclusions}

As the first generation of cardiac regenerative therapy, many clinical trials of cell injection therapy have been already performed. The controversial arguments about its effectiveness will be settled in next several years. Tissue engineered myocardial patches have now emerged as the second generation and previous studies indicate promising potential for rescuing damaged heart. As the third generation, tissue-engineered pulsatile myocardial tissues should support heart contraction physically. Furthermore, future development of cell sourcing and scaling-up technologies may realize "bioengineered hearts".

\section{Acknowledgment}

This work is granted by the Japan Society for the Promotion of Science (JSPS) through the "Funding Program for World-Leading Innovative R\&D on Science and Technology (FIRST Program)," initiated by the Council for Science and Technology Policy (CSTP).

\section{References}

Asahara, T., T. Murohara, et al. (1997). Isolation of putative progenitor endothelial cells for angiogenesis. Science 275(5302): 964-967.

Beltrami, A. P., L. Barlucchi, et al. (2003). Adult cardiac stem cells are multipotent and support myocardial regeneration. Cell 114(6): 763-776.

Bergmann, O., R. D. Bhardwaj, et al. (2009). Evidence for cardiomyocyte renewal in humans. Science 324(5923): 98-102.

Birla, R. K., V. Dhawan, et al. (2009). Cardiac cells implanted into a cylindrical, vascularized chamber in vivo: pressure generation and morphology. Biotechnol Lett 31(2): 191201.

Chachques, J. C., J. C. Trainini, et al. (2007). Myocardial assistance by grafting a new bioartificial upgraded myocardium (MAGNUM clinical trial): one year follow-up. Cell Transplant 16(9): 927-934. 
Haraguchi, Y., T. Shimizu, et al. (2006). Electrical coupling of cardiomyocyte sheets occurs rapidly via functional gap junction formation. Biomaterials 27(27): 4765-4774.

Hata, H., G. Matsumiya, et al. (2006). Grafted skeletal myoblast sheets attenuate myocardial remodeling in pacing-induced canine heart failure model. J Thorac Cardiovasc Surg 132(4): 918-924.

Hida, N., N. Nishiyama, et al. (2008). Novel cardiac precursor-like cells from human menstrual blood-derived mesenchymal cells. Stem Cells 26(7): 1695-1704.

Hofmann, M., K. C. Wollert, et al. (2005). Monitoring of bone marrow cell homing into the infarcted human myocardium. Circulation 111(17): 2198-2202.

Itabashi, Y., S. Miyoshi, et al. (2005). A new method for manufacturing cardiac cell sheets using fibrin-coated dishes and its electrophysiological studies by optical mapping. Artif Organs 29(2): 95-103.

Ito, A., E. Hibino, et al. (2005). Construction and delivery of tissue-engineered human retinal pigment epithelial cell sheets, using magnetite nanoparticles and magnetic force. Tissue Eng 11(3-4): 489-496.

Kitabayashi, K., A. Siltanen, et al. (2010). Bcl-2 expression enhances myoblast sheet transplantation therapy for acute myocardial infarction. Cell Transplant 19(5): 573588.

Kobayashi, H., T. Shimizu, et al. (2008). Fibroblast sheets co-cultured with endothelial progenitor cells improve cardiac function of infarcted hearts. J Artif Organs 11(3): 141-147.

Kofidis, T., J. L. de Bruin, et al. (2004). Injectable bioartificial myocardial tissue for large-scale intramural cell transfer and functional recovery of injured heart muscle. $J$ Thorac Cardiovasc Surg 128(4): 571-578.

Kofidis, T., A. Lenz, et al. (2003). Pulsatile perfusion and cardiomyocyte viability in a solid three-dimensional matrix. Biomaterials 24(27): 5009-5014.

Kondoh, H., Y. Sawa, et al. (2006). Longer preservation of cardiac performance by sheetshaped myoblast implantation in dilated cardiomyopathic hamsters. Cardiovasc Res 69(2): 466-475.

Kubo, H., T. Shimizu, et al. (2007). Creation of myocardial tubes using cardiomyocyte sheets and an in vitro cell sheet-wrapping device. Biomaterials 28(24): 3508-3516.

Lee, S. T., A. J. White, et al. (2011). Intramyocardial injection of autologous cardiospheres or cardiosphere-derived cells preserves function and minimizes adverse ventricular remodeling in pigs with heart failure post-myocardial infarction. J Am Coll Cardiol 57(4): 455-465.

Leor, J., S. Aboulafia-Etzion, et al. (2000). Bioengineered cardiac grafts: A new approach to repair the infarcted myocardium? Circulation 102(19 Suppl 3): III56-61.

Li, R. K., Z. Q. Jia, et al. (1999). Survival and function of bioengineered cardiac grafts. Circulation 100(19 Suppl): II63-69.

Martin-Rendon, E., S. J. Brunskill, et al. (2008). Autologous bone marrow stem cells to treat acute myocardial infarction: a systematic review. Eur Heart J 29(15): 1807-1818.

Matsuura, K., A. Honda, et al. (2009). Transplantation of cardiac progenitor cells ameliorates cardiac dysfunction after myocardial infarction in mice. J Clin Invest 119(8): 22042217. 
Memon, I. A., Y. Sawa, et al. (2005). Repair of impaired myocardium by means of implantation of engineered autologous myoblast sheets. J Thorac Cardiovasc Surg 130(5): 1333-1341.

Menasche, P., O. Alfieri, et al. (2008). The Myoblast Autologous Grafting in Ischemic Cardiomyopathy (MAGIC) trial: first randomized placebo-controlled study of myoblast transplantation. Circulation 117(9): 1189-1200.

Menasche, P., A. A. Hagege, et al. (2001). Myoblast transplantation for heart failure. Lancet 357(9252): 279-280.

Mirsadraee, S., H. E. Wilcox, et al. (2006). Development and characterization of an acellular human pericardial matrix for tissue engineering. Tissue Eng 12(4): 763-773.

Miyagawa, S., A. Saito, et al. (2010). Impaired myocardium regeneration with skeletal cell sheets--a preclinical trial for tissue-engineered regeneration therapy. Transplantation 90(4): 364-372.

Miyagawa, S., Y. Sawa, et al. (2005). Tissue cardiomyoplasty using bioengineered contractile cardiomyocyte sheets to repair damaged myocardium: their integration with recipient myocardium. Transplantation 80(11): 1586-1595.

Miyahara, Y., N. Nagaya, et al. (2006). Monolayered mesenchymal stem cells repair scarred myocardium after myocardial infarction. Nat Med 12(4): 459-465.

Morritt, A. N., S. K. Bortolotto, et al. (2007). Cardiac tissue engineering in an in vivo vascularized chamber. Circulation 115(3): 353-360.

Oh, H., S. B. Bradfute, et al. (2003). Cardiac progenitor cells from adult myocardium: homing, differentiation, and fusion after infarction. Proc Natl Acad Sci U S A 100(21): 12313-12318.

Okano, T., N. Yamada, et al. (1993). A novel recovery system for cultured cells using plasma-treated polystyrene dishes grafted with poly(N-isopropylacrylamide). $J$ Biomed Mater Res 27(10): 1243-1251.

Opie, S. R. and N. Dib (2006). Surgical and catheter delivery of autologous myoblasts in patients with congestive heart failure. Nat Clin Pract Cardiovasc Med 3 Suppl 1: S4245.

Ott, H. C., T. S. Matthiesen, et al. (2008). Perfusion-decellularized matrix: using nature's platform to engineer a bioartificial heart. Nat Med 14(2): 213-221.

Papadaki, M., N. Bursac, et al. (2001). Tissue engineering of functional cardiac muscle: molecular, structural, and electrophysiological studies. Am J Physiol Heart Circ Physiol 280(1): H168-178.

Psaltis, P. J., A. C. Zannettino, et al. (2008). Concise review: mesenchymal stromal cells: potential for cardiovascular repair. Stem Cells 26(9): 2201-2210.

Puceat, M. (2008). Pharmacological approaches to regenerative strategies for the treatment of cardiovascular diseases. Curr Opin Pharmacol 8(2): 189-192.

Radisic, M., H. Park, et al. (2007). Biomimetic approach to cardiac tissue engineering. Philos Trans R Soc Lond B Biol Sci 362(1484): 1357-1368.

Sekine, H., T. Shimizu, et al. (2008). Endothelial cell coculture within tissue-engineered cardiomyocyte sheets enhances neovascularization and improves cardiac function of ischemic hearts. Circulation 118(14 Suppl): S145-152.

Sekine, H., T. Shimizu, et al. (2006). Cardiomyocyte bridging between hearts and bioengineered myocardial tissues with mesenchymal transition of mesothelial cells. J Heart Lung Transplant 25(3): 324-332. 
Sekine, H., T. Shimizu, et al. (2006). Pulsatile myocardial tubes fabricated with cell sheet engineering. Circulation 114(1 Suppl): I87-93.

Sekiya, N., G. Matsumiya, et al. (2009). Layered implantation of myoblast sheets attenuates adverse cardiac remodeling of the infarcted heart. J Thorac Cardiovasc Surg 138(4): 985-993.

Sekiya, S., T. Shimizu, et al. (2006). Bioengineered cardiac cell sheet grafts have intrinsic angiogenic potential. Biochem Biophys Res Commun 341(2): 573-582.

Shimizu, T., H. Sekine, et al. (2006). Long-term survival and growth of pulsatile myocardial tissue grafts engineered by the layering of cardiomyocyte sheets. Tissue Eng 12(3): 499-507.

Shimizu, T., H. Sekine, et al. (2009). Cell sheet-based myocardial tissue engineering: new hope for damaged heart rescue. Curr Pharm Des 15(24): 2807-2814.

Shimizu, T., H. Sekine, et al. (2006). Polysurgery of cell sheet grafts overcomes diffusion limits to produce thick, vascularized myocardial tissues. FASEB J 20(6): 708-710.

Shimizu, T., M. Yamato, et al. (2002). Electrically communicating three-dimensional cardiac tissue mimic fabricated by layered cultured cardiomyocyte sheets. J Biomed Mater Res 60(1): 110-117.

Shimizu, T., M. Yamato, et al. (2002). Fabrication of pulsatile cardiac tissue grafts using a novel 3-dimensional cell sheet manipulation technique and temperature-responsive cell culture surfaces. Circ Res 90(3): e40.

Shimizu, T., M. Yamato, et al. (2003). Cell sheet engineering for myocardial tissue reconstruction. Biomaterials 24(13): 2309-2316.

Shin, M., O. Ishii, et al. (2004). Contractile cardiac grafts using a novel nanofibrous mesh. Biomaterials 25(17): 3717-3723.

Siltanen, A., K. Kitabayashi, et al. (2011). hHGF Overexpression in Myoblast Sheets Enhances Their Angiogenic Potential in Rat Chronic Heart Failure. PLoS One 6(4): e19161.

Tan, M. Y., W. Zhi, et al. (2009). Repair of infarcted myocardium using mesenchymal stem cell seeded small intestinal submucosa in rabbits. Biomaterials 30(19): 3234-3240.

Vunjak-Novakovic, G., N. Tandon, et al. (2010). Challenges in cardiac tissue engineering. Tissue Eng Part B Rev 16(2): 169-187.

Wollert, K. C. (2008). Cell therapy for acute myocardial infarction. Curr Opin Pharmacol 8(2): 202-210.

Yildirim, Y., H. Naito, et al. (2007). Development of a biological ventricular assist device: preliminary data from a small animal model. Circulation 116(11 Suppl): I16-23.

Yuasa, S., Y. Itabashi, et al. (2005). Transient inhibition of BMP signaling by Noggin induces cardiomyocyte differentiation of mouse embryonic stem cells. Nat Biotechnol 23(5): 607-611.

Zhang, M., D. Methot, et al. (2001). Cardiomyocyte grafting for cardiac repair: graft cell death and anti-death strategies. J Mol Cell Cardiol 33(5): 907-921.

Zhu, W., I. Shiojima, et al. (2008). IGFBP-4 is an inhibitor of canonical Wnt signalling required for cardiogenesis. Nature 454(7202): 345-349.

Zimmermann, W. H. (2011). Embryonic and embryonic-like stem cells in heart muscle engineering. J Mol Cell Cardiol 50(2): 320-326.

Zimmermann, W. H. and R. Cesnjevar (2009). Cardiac tissue engineering: implications for pediatric heart surgery. Pediatr Cardiol 30(5): 716-723. 
Zimmermann, W. H., M. Didie, et al. (2006). Heart muscle engineering: an update on cardiac muscle replacement therapy. Cardiovasc Res 71(3): 419-429.

Zimmermann, W. H., I. Melnychenko, et al. (2006). Engineered heart tissue grafts improve systolic and diastolic function in infarcted rat hearts. Nat Med 12(4): 452-458.

Zimmermann, W. H., K. Schneiderbanger, et al. (2002). Tissue engineering of a differentiated cardiac muscle construct. Circ Res 90(2): 223-230. 


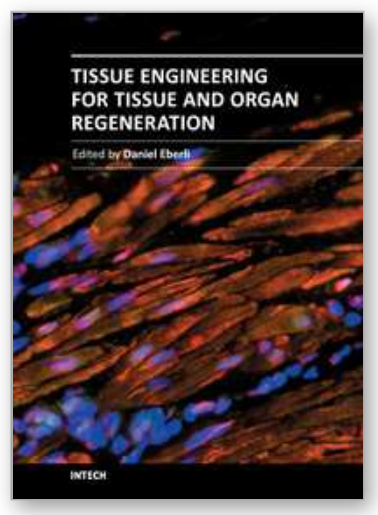

\author{
Tissue Engineering for Tissue and Organ Regeneration \\ Edited by Prof. Daniel Eberli
}

ISBN 978-953-307-688-1

Hard cover, 454 pages

Publisher InTech

Published online 17, August, 2011

Published in print edition August, 2011

Tissue Engineering may offer new treatment alternatives for organ replacement or repair deteriorated organs. Among the clinical applications of Tissue Engineering are the production of artificial skin for burn patients, tissue engineered trachea, cartilage for knee-replacement procedures, urinary bladder replacement, urethra substitutes and cellular therapies for the treatment of urinary incontinence. The Tissue Engineering approach has major advantages over traditional organ transplantation and circumvents the problem of organ shortage. Tissues reconstructed from readily available biopsy material induce only minimal or no immunogenicity when reimplanted in the patient. This book is aimed at anyone interested in the application of Tissue Engineering in different organ systems. It offers insights into a wide variety of strategies applying the principles of Tissue Engineering to tissue and organ regeneration.

\title{
How to reference
}

In order to correctly reference this scholarly work, feel free to copy and paste the following:

Tatsuya Shimizu (2011). Myocardial Tissue Engineering, Tissue Engineering for Tissue and Organ Regeneration, Prof. Daniel Eberli (Ed.), ISBN: 978-953-307-688-1, InTech, Available from: http://www.intechopen.com/books/tissue-engineering-for-tissue-and-organ-regeneration/myocardial-tissueengineering

\section{INTECH}

open science | open minds

\author{
InTech Europe \\ University Campus STeP Ri \\ Slavka Krautzeka 83/A \\ 51000 Rijeka, Croatia \\ Phone: +385 (51) 770447 \\ Fax: +385 (51) 686166 \\ www.intechopen.com
}

\author{
InTech China \\ Unit 405, Office Block, Hotel Equatorial Shanghai \\ No.65, Yan An Road (West), Shanghai, 200040, China \\ 中国上海市延安西路65号上海国际贵都大饭店办公楼 405 单元 \\ Phone: +86-21-62489820 \\ Fax: +86-21-62489821
}


(C) 2011 The Author(s). Licensee IntechOpen. This chapter is distributed under the terms of the Creative Commons Attribution-NonCommercialShareAlike-3.0 License, which permits use, distribution and reproduction for non-commercial purposes, provided the original is properly cited and derivative works building on this content are distributed under the same license. 